\title{
Research and implementation of medical image processing technology
}

\author{
Wang Jinguo ${ }^{1, a}$, Wang $\mathrm{Na}^{2, b^{*}}$ (corresponding author), Wang Rui, ${ }^{3, \mathrm{c}}$ \\ ${ }^{1}$ Department of Urology, the First Hospital of Jilin University,China \\ ${ }^{2}$ Department of Anesthesiology, the First Hospital of Jilin University, China \\ ${ }^{3}$ Department of Information Engineering, Jilin Business and Technology College.

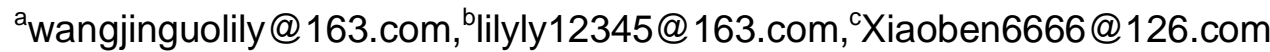

\begin{abstract}
Keywords: PACS. Medical image processing. Image segmentation. Visualization. VKT
Abstract. Picture archiving and communications system (PACS) has become an important tool for the analysis and processing of medical image information, and it is also the basic element of the construction of digital hospital. In PACS, the image processing module is very important, and the research and implementation of high performance image processing module can enhance the image processing effect, improve the work efficiency and improve medical diagnosis level. In this paper, the key technology of medical image processing in PACS system is realized, which can realize the function of the image file of medical digital image and communication standard DICMO.
\end{abstract}

\section{Introduction}

In recent years, many large and medium-sized hospitals in China, with the progress of science and technology and social development, more and more attention to the construction of information technology, the concept of digital hospital is proposed[1]. The digital hospital uses computer technology, multimedia equipment, network and other information technology to integrate the patient information, health economic information and hospital information, stored in the entire community health care database, so that patients in any network can query the personal health records, but also online registration and appointment[2]. At the same time, with the rapid development of the digital hospital, medical image is increasing, so we have to use the computer to store these images, and to carry out the corresponding image processing[3]. In 1980s, the concept of PACS was proposed, because of the rapid development of digital imaging devices such as CT, etc., which makes the medical image information system can communicate with other systems gradually, which is composed of the hospital information system[4].

A typical hospital information system mainly consists of five parts, the 5 part mainly includes: picture archiving and communication systems(PACS), clinical information system( CIS ), radiology information system( RIS ), hospital information system( HIS ), laboratory information system( LIS ). PACS is one of the important modules. PACS is a DICOM3.0 international standard design, with high performance server, network and storage device to constitute the hardware support platform, with large relational database as data and image storage management tool, the medical image collection, transmission, storage and diagnosis as the core, is set image acquisition and storage management, image diagnosis and management, integrated information management, integrated application system[6]. Its main task is to radiology department daily producing various kinds of medical images, including images of MRI, CT, ultrasound, X-ray equipment through DICOM 3.0 standard international interface to digital mass stored. When needed in the authorized to soon to use, 
while adding some auxiliary diagnosis management function[7].

\section{DICOM technology introduction}

DICOM(Digital Imaging and Communications in Medicine) DICOM, the digital image transmission protocol, is proposed by the United States Association of the United States of America and the American Association of electrical appliances. It defines the medical image format used in the processing, storage, printing and transmission of medical images generated by different devices. DICOM standard is the development of information technology, a variety of high-tech medical equipment and hospital information system, especially the development of PACS and remote medical system. Because technology development we can easily obtain the sequence of the organ's images, but the format of the DICOM image is not consistent with the medical device model. As the format is not consistent with a lot of questions: how do these different images are stored and managed? How to interconnect and transmit images between different devices? Obviously, to solve these problems, all of the medical devices produced by the image must comply with a unified standard.

\section{Dicom file format}

DICOM file is divided into two parts: header and datasets. DICOM file is the DICOM file header, file header contains: file order, prefix and header information. To maintain compatibility with the general file structure, the 128 byte of the hexadecimal component files. The prefix part is a file identifier of the character "DICM", which identifies the file as the DICOM file. Header information is by the data identifier (tag) in the group for Ox0002 DICOM data elements that constitute the data set header information includes the formation of DICOM files when the transfer syntax and other information. Data set is an instance of the information object. It is a collection of data elements, which is used to store the information object definition (OID). Data element is the most basic unit of DICOM file, and each data element corresponds to one of the information, which is the attribute value of the information object corresponding to the data set. Data element is composed of four parts: tag, VR (Representation Value), value length, and data value.

\section{The design and implementation of CT image 3D visualization}

Medical image 3D visualization part of the processing object is in line with the DICOM standard CT or MRI images, the main realization of the CT images of the surface rendering and volume rendering, providing dynamic window width, window position adjustment, three-dimensional body rotation, scaling, translation control and body data preprocessing and other functions. Sequences CT or MRI images are a set of multiple images obtained by the human body scanning. They are characterized by parallel and regular in the space position. The pixel points of the image are arranged in a regular grid. In the process of research and development of software, we use two kinds of algorithms: the surface rendering and volume rendering to reconstruct multiple CT images. We find that the surface rendering is a small part of the data field, which has a fast speed, and can be fast and flexible to rotate and transform the illumination effect. However, due to the large dependence on surface reconstruction, the accuracy of segmentation is very high, so the shape of the soft tissue is not obvious, the brightness changes is small, and the three-dimensional display of blood vessels is not satisfactory. Moreover, surface rendering can not preserve the integrity of the data, the reconstruction of the object surface and lost the internal information. Volume rendering is the use of all data, through the processing of the three-dimensional data field in each voxel 
separately, and synthetic images with three-dimensional effect. Volume rendering has good effect on the three-dimensional display of the tissue and organs of the shape feature. But in the process of rendering the original body, it is usually to traverse each individual element in the body data field, and thus the amount of calculation is large, and the speed of image imaging is slow. When changing illumination and viewpoint, the projection operation is to be performed, so the speed of interaction is slower. Therefore, in order to meet the requirements of different applications, we consider the design of the system while providing two visualization methods.

Aiming at the problem of noise in the sequence images of the input, the system must provide the function of the whole body data field to realize the noise reduction. Similar to 2D image processing, 3D data field can also be used to realize the enhancement of 3D data field by the window width and window level adjustment. We provide the function of slice interpolation. The general input section provides the information of cross section. Then, we can use the method of computer graphics to obtain the information of arbitrary cutting surface. Also although object visualization system is based on the spatial parallel serial CT or MRI images, but for the rotational speed of image sequences also provide 3D visualization function. This function implementation can use pictures of continuous playback technology, this technology the Hully with rotation technique shooting of such as vascular according to images of three-dimensional visual effect is better.

\section{Experimental results}

The system basically completed the design of the function. By running the test in the actual PACS system, the results show that the image processing module in this paper has a high operating efficiency and good openness.

\section{References}

[1] Hussein R, Engelmann U, Schroeter A, et al. DICOM structured reporting Part 2. Problems and challenges in implementation for PACS workstations1[J]. Radiographics, 2004, 24(3): 897-909.

[2] Grauer D, Cevidanes L S H, Proffit WR. Working with DICOM craniofacial images[J].American Journal of Orthodontics and Dentofacial Orthopedics, 2009, 136(3): 460-470.

[3] Fernàndez-Bayó J, Barbero O,Rubies C,et al. Distributing Medical Images with Internet Technologies : A DICOM Web Server and a DICOM Java Viewer1[J]. Radiographics, 2000, 20(2): 581-590.

[4] Can A, H Shen, J N Turner, et al, Rapid Automated Tracing and Feature Extraction from Live High-Resolution Retinal Fundus Images Using Direct Exploratory Algorithms, IEEE Transactions on Information Technology in Biomedicine, 1999, 3(2): 125 138.

[5] M Sonka, M D Winniford, X Zhang, et al, Robust Simultaneous Detection of Coronary Borders in Complex Images, IEEE Transaction on Medical Images, 1995, 14(1): 151 161.

[6] McCormick B H, DeFanti T A, Brown M D, Visualization in Scientific Computing, Computer Graphics, 1987, 21(6): 249 266. 
